\title{
Captured at millimeter wavelengths: a flare from the classical T Tauri star DQ Tauri
}

\author{
D. M. Salter ${ }^{1}$, M. R. Hogerheijde ${ }^{1}$, and G. A. Blake $^{2}$ \\ ${ }^{1}$ Leiden Observatory, Leiden University, PO Box 9513, 2300 RA Leiden, The Netherlands \\ e-mail: [demerese; michiel]@strw. leidenuniv.nl \\ 2 California Institute of Technology, Division of Geological and Planetary Sciences, Mail Stop 150-21, Pasadena, CA 91125, USA \\ e-mail: gab@gps.caltech.edu
}

Received 14 August 2008 / Accepted 21 October 2008

\section{ABSTRACT}

\begin{abstract}
For several hours on 2008 April 19, the T Tauri spectroscopic binary DQ Tau was observed to brighten, reaching a maximum detected flux of $468 \mathrm{mJy}$ and likely making it (briefly) the brightest object at $3 \mathrm{~mm}$ in the Taurus star-forming region. We present the light curve of a rarely before observed millimeter flare originating in the region around a pre-main-sequence star, and the first from a classical $\mathrm{T}$ Tauri star. We discuss the properties and nature of the flaring behavior in the context of pulsed accretion flows (the current picture based largely on studies of this object's optically variable spectrum), as well as magnetospheric re-connection models (a separate theory that predicts millimeter flares for close binaries of high orbital eccentricity). We believe that the flare mechanism is linked to the binary orbit, and therefore periodic. DQ Tau makes a strong case for multi-wavelength follow-up studies, performed in parallel, of future flares to help determine whether magnetospheric and dynamical interactions in a proto-binary system are independent.
\end{abstract}

Key words. radio continuum: stars - stars: individual: DQ Tau - stars: pre-main sequence - stars: binaries: spectroscopic stars: flare - stars: magnetic fields

\section{Introduction}

Observations of pre-main-sequence (PMS) stars at millimeter wavelengths are used to study thermal emission from gas and dust in their circumstellar disks where planets may be forming. At these wavelengths, the disks are optically thin and the integrated flux is related directly to the total amount of cold, circumstellar material. But on short timescales, we show how the dominating mechanism for millimeter emission can shift to high-energy, short-period processes. We present $3 \mathrm{~mm}$ continuum observations of the low-mass, classical T Tauri star (CTTS) DQ Tau, which was observed to brighten for many hours on 2008 April 19, reaching a maximum detected flux of $468 \mathrm{mJy}$ (compared to $17 \mathrm{mJy}$ in the days both before and after the event).

A stellar flare at these typically quiescent wavelengths, like a solar flare in the millimeter, is believed to be the superposition of a gyro-synchrotron spectrum with that from synchrotron emission, the latter peaking in the sub-millimeter to far-infrared (Kaufmann et al. 1986). The first light curve for a strong millimeter flare observed toward a young stellar object (YSO), GMR-A in Orion, was reported by Bower et al. (2003) and attributed to magnetic activity. In protostellar environments, scenarios where magnetic fields can interact, re-connect, and accelerate electrons to high energies are thought to include single-star stellar flares as we see in the Sun, interactions due to changes in the magnetic field lines between a star and its accretion disk, and colliding magnetospheres and coronal flares between components of a multiple-star system.

While longer-term radio variability is well documented in YSOs, and can also be the result of magnetic activity like starspots, few outbursts have been observed (Feigelson \& Montmerle 1999). DQ Tau is only the fourth flare detected toward a PMS object, and the first from a CTTS. In addition to GMR-A, our observation follows the re-occurring flares from the weak-line T Tauri star (WTTS) V773 Tau A, studied in depth by Massi et al. (2002, 2006, 2008), and a single $1.3 \mathrm{~cm}$ flare from a second YSO in Orion named ORBS by Forbrich et al. (2008).

In Orion, follow-up study of GMR-A confirmed a photospheric origin of gyro-synchrotron emission around a single magnetically-active WTTS (Furuya et al. 2003), whereas observations of the deeply embedded ORBS could only limit the emitting region to a radius $\leq 2 \mathrm{AU}$ (Forbrich et al. 2008). In contrast, the periodic flaring from V773 Tau A is the first extrasolar evidence of interacting coronal flares, attributed in this case to collisions near periastron between extended helmet streamers from the binary components (Massi et al. 2008), a phenomenon closely analogous to the situation for DQ Tau. With a higher orbital eccentricity ( $e=0.556$, vs. 0.3 for V773 Tau A), the DQ Tau system geometry predicts an overlap of its two protostellar magnetospheres at each periastron passage without the need for extended structure (Mathieu et al. 1997).

\section{The DQ Tau system}

Mathieu et al. (1997) found DQ Tau ( $\alpha=04: 46: 53.06$, $\delta=+17: 00: 00.1)$ to be a non-eclipsing, double-lined spectroscopic binary, comprised of two relatively equal-mass stars $\left(\sim 0.65 M_{\odot}\right)$ with spectral types in the range of $\mathrm{K} 7$ to $\mathrm{M} 1$ and a robust orbital period of 15.804 days. At closest approach, the binary separation is $8 R_{*}\left(=13 R_{\odot}\right)$, roughly four times smaller than the $30 \mathrm{R}_{*}\left(=60 R_{\odot}\right)$ separation for V773 Tau A, and less than twice the expected $\mathrm{T}$ Tauri stellar magnetosphere size of $\sim 5 R_{*}$ (Shu et al. 1994; Hartmann et al. 1994). As a result of 
the binary geometry and magnetospheric models, re-connection events have been predicted for this source (Mathieu et al. 1997), but never tested observationally. We provide evidence that this effect is occurring and that it may be repeatable and relevant to many similar T Tauri systems.

The spectral energy distribution (SED) of DQ Tau is fairly typical of a CTTS. It is best fit by Mathieu et al. (1997) using a large circumbinary disk of about $0.002-0.02 M_{\odot}$. They find that an additional $5 \times 10^{-10} M_{\odot}$ of warm $(\sim 1000 \mathrm{~K})$ circumstellar material close to the stars is necessary to explain the near-infrared excess, but not enough to maintain the observed accretion rate of $5 \times 10^{-8} M_{\odot} \mathrm{yr}^{-1}$ (see Hartigan et al. 1995). DQ Tau must be transporting material into its inner regions. Both a broad bright $10 \mu \mathrm{m}$ silicate feature (see Furlan et al. 2006), and the absence of strongly blocked forbidden-line transitions in the receding stellar flow, support optically thin material in a region up to $0.4 \mathrm{AU}$ around the binary (Basri et al. 1997; Najita et al. 2003).

At optical wavelengths, the system is known to brighten by $0.5 \mathrm{mag}$, coinciding with periastron, either shortly before or at closest approach. The mechanism regulating the optical activity is variable, as brightenings have been observed for only $65 \%$ of periastron encounters, and in isolated events, multiple brightenings were seen in a single orbit (Mathieu et al. 1997). All brightenings were recorded within an orbital phase window of 0.30 (or 5 days) centered on a phase $(\Phi)$ of 0.9 , where periastron defines $\Phi=0.0,1.0$ (Mathieu et al. 1997). This evidence for accretion presents an additional puzzle, indeed for most binaries, since a large inner gap is expected to be cleared by the resonant and tidal forces generated by stellar movements, thereby quickly isolating a circumbinary disk from one or two circumstellar disks. The small binary separation of DQ Tau, however, reduces the likelihood of stable circumstellar disks. These effects combined should speed up disk dissipation and halt accretion processes. Instead, it is unclear how easily material can cross the predicted gap and why some binaries show inner cleared regions and others, like DQ Tau, do not (Jensen et al. 2007).

In this context, DQ Tau was the first system to be studied in terms of pulsed accretion flows (Mathieu et al. 1997). Extensive observations of the system's optical variability and photospheric line properties show increased veiling effects, spectral bluenings, emergence of outflow signatures in spectral lines, and the double-line structure in Ca II suggesting that bright blueshifted material is being emitted near the surface of the star during an event (Basri et al. 1997). All these effects at periastron are consistent with pulsed accretion flows based on the theoretical models of Artymowicz \& Lubow (1996) that describe how the eccentric binary orbit periodically perturbs the outer disk, causing material to collect at stable points near the inner rim and then to stream across the gap. However, the theory does not require the peak accretion rate to occur near periastron. Instead it has been postulated that the accretion mechanism is further regulated by strongly variable magnetic fields (Mathieu et al. 1997).

\section{CARMA observations}

We observed DQ Tau at $115 \mathrm{GHz}(\approx 2.7 \mathrm{~mm})$ with the Combined Array for Research in Millimeter-wave Astronomy (CARMA) during 5 tracks in April 2008, totaling $4 \mathrm{~h}$ on-source. The millimeter array, located in eastern California (USA), provides 150 baselines of $30-350 \mathrm{~m}$ in its $\mathrm{C}$ configuration and records one linear polarization. Our program cycled through 5 Taurus sources during each track, scanning each object for 12 min and obtaining 1-6 scans per source, depending on the track length. A 5-min scan of the gain calibrator 3C 111 was included every
Table 1. DQ Tau flux and intensity data at $2.7 \mathrm{~mm}(115 \mathrm{GHz})$.

\begin{tabular}{ccc}
\hline \hline $\mathrm{JD}^{(2454500+)^{a}}$ & ${\text { Intensity }\left(\mathrm{mJy} \mathrm{beam}^{-1}\right)}^{\text {Flux }_{(\mathrm{mJy})^{b}}}$ \\
\hline 76.3330 & $37.4 \pm 15.0^{c}$ & - \\
76.3923 & $345.0 \pm 19.8$ & 419 \\
76.4534 & $355.1 \pm 11.4$ & 468 \\
76.5132 & $216.8 \pm 9.2$ & 284 \\
76.5789 & $150.3 \pm 6.1$ & 194 \\
\hline Quiescent & $13.3 \pm 1.5$ & 17
\end{tabular}

${ }^{a}$ Indicates the start of the 12-min scan. ${ }^{b}$ Determined by a Gaussian fit to the continuum image. ${ }^{c}$ Data point suffers from severe de-correlation at longer baselines.

12-24 min. Multiple observing scripts allowed rotation of the source order in each track so that we could achieve more efficient $(u, v)$-coverage per source. In addition to the phase calibration using 3C 111, we observed the planet Uranus for flux calibration at the start of each track, as well as the radio source 3C 84 for passband calibration.

All data were processed using the MIRIAD data reduction software program, optimized for CARMA. We calibrated each track separately before combining the data into a final $(u, v)$-averaged continuum image. In the inversion step a natural weighting for the $(u, v)$-coverage was applied, and cleaning was performed down to a cutoff of $2 \sigma$. The continuum maps are a composite of two $500 \mathrm{MHz}$ bands covering the frequency ranges 110.94-111.41 GHz and $114.80-115.27 \mathrm{GHz}$, corresponding to the lower and upper sidebands, respectively.

\section{Results and analysis}

In the combined and calibrated DQ Tau image ( $3 \mathrm{~h}$ on-source) we achieved a sky rms noise level of $1.45 \mathrm{mJy} \mathrm{beam}^{-1}$ and a beam size of $1.92^{\prime \prime} \times 0.99^{\prime \prime}$ toward the Taurus star-forming region, corresponding to a spatial resolution of $\sim 140-270$ AU at a distance of $140 \mathrm{pc}$. Since we found that the source was considerably brighter on 19 April than during any other observation date (Fig. 1), data for that track were not included in this final continuum image. And thus the quiescent values for the other nights combined then give a peak intensity of $13.3 \mathrm{mJy} \mathrm{beam}^{-1}$ and a flux, determined by a Gaussian fit, of $17 \mathrm{mJy}$.

Review of the unexpected result from 19 April revealed that the brightening was a real effect. Many instrumental and data processing errors could be ruled out because none of the 4 other sources exhibited a similar effect, the brightening persisted throughout 5 source cycles in an 8-h period, and, the same observing script was used for 3 of the 5 tracks. The location of the peak emission at the time of the flare is consistent with the coordinates of the binary on all nights and therefore no background source is suspected. Finally, while the linear feeds do rotate while tracking the source, we expect a flare mimicked by the presence of strong polarization would not only be present in the nearly identical tracks of the adjacent days, but that the emission profile would be more symmetric about its peak.

To understand the nature of the DQ Tau brightening, we reduced each of the track's five 12-min source scans separately to produce individual continuum maps. We then plotted the maximum source intensity (Jy beam ${ }^{-1}$ of the peak pixel) as a function of time (Fig. 1). DQ Tau clearly exhibited a flaring behavior, rising to a maximum brightness in $\sim 1-2 \mathrm{~h}$, coinciding with the start of our track, and shortly thereafter, decaying (roughly exponentially) throughout the remainder of the observation, returning to half the observed peak intensity within a 2-h period. 


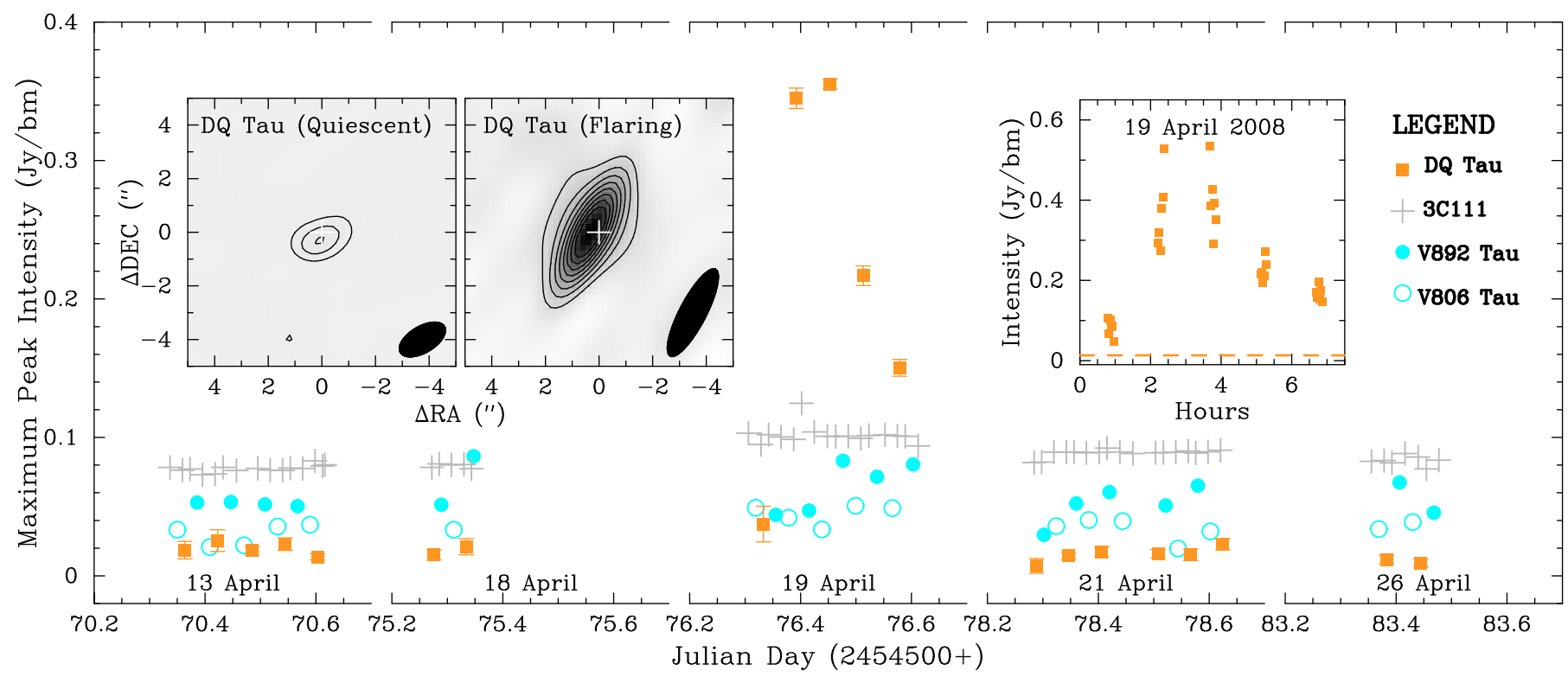

Fig. 1. A plot of the maximum intensity of each scan as a function of Julian Day. Filled squares represent DQ Tau, circles the 2 brightest Taurus sources in our tracks, and crosses are the calibrator (divided by a factor 50). Error bars for DQ Tau are the sky rms for each scan. Upper left inset: continuum images of DQ Tau at $2.7 \mathrm{~mm}(115 \mathrm{GHz})$. White crosses indicate the 2MASS infrared position. Pixels are $0.2^{\prime \prime} \times 0.2^{\prime \prime}$ and the same grayscale is used for both images (ranging $-3.14 \times 10^{-2}$ to $3.55 \times 10^{-1} \mathrm{Jy} \mathrm{beam}^{-1}$ ). On the left, the combined image from the 4 quiescent tracks. Contours are drawn at $3 \sigma$ levels where $\sigma=1.45 \times 10^{-3}$. At right, DQ Tau brightens by a factor of 27 on 2008 April $19(\mathrm{JD}=2454576.4534)$, peaking at $355 \mathrm{mJy}_{\text {beam }}^{-1}$. We determine a flux of $468 \mathrm{mJy}$ for this scan. Upper right inset: the intensity (Jy beam ${ }^{-1}$ ) of the peak pixel at the location of DQ Tau throughout the track on 19 April, now sub-divided into 2-min intervals. The horizontal dashed line indicates the maximum intensity found for the quiescent image. The second and third scans show sharply increasing and decreasing intensity, respectively.

In Fig. 1 we show the results of performing the same intensity analysis on the individual scans for three additional sources (our two brightest Taurus sources plus the gain calibrator). These sources appear constant in flux for all observations (distributed over 13 days). The same is not true for DQ Tau, which remains unresolved throughout its brightening event. At peak brightness, its flux during our third scan on 19 April $(\mathrm{JD}=2454576.4534)$ was $468 \mathrm{mJy}$, roughly 27 times the quiescent value (Table 1), likely making DQ Tau the brightest object at $3 \mathrm{~mm}$ in Taurus at the time of our observation if we consider that the class-defining source T Tau is just $56 \pm 10 \mathrm{mJy}$ at $3 \mathrm{~mm}$ (Ohashi et al. 1996) and the bright, extended source HL Tau is $100 \mathrm{mJy}$ (Beckwith et al. 1986). Finally, the flare is in stark contrast to (single-dish) measurements of DQ Tau that find $125 \mathrm{mJy}$ at $1 \mathrm{~mm}$ and $91 \mathrm{mJy}$ at $1.3 \mathrm{~mm}$ (Beckwith \& Sargent 1991; Beckwith et al. 1990). Our quiescent value of $17 \mathrm{mJy}$, on the other hand, is a better fit to the expected millimeter spectral slope.

The true shape of the flare onset and its exact peak are less precise. Larger error bars for this date are a result of significant flagging due to strong decorrelation on longer baselines, particularly during the first half of the track. The gain calibrator, which has an average flux of $3.7 \mathrm{Jy}$ for each track, has a larger standard deviation $(\sigma)$ for the maximum intensity of each 5-min calibrator scan on the flare date $\left(0.257 \mathrm{Jy} \mathrm{bm}^{-1}\right)$ than, for example, the longest tracks on 13 April $\left(0.095 \mathrm{Jy} \mathrm{bm}^{-1}\right)$ and 21 April $\left(0.103 \mathrm{Jy} \mathrm{bm}^{-1}\right)$. These effects do not compromise the validity of the flare detection. For comparison, the two brightest Taurus sources observed have a $\sigma$ of $0.013 \mathrm{Jy} \mathrm{bm}^{-1}$ and $0.006 \mathrm{Jy} \mathrm{bm}^{-1}$ for their five 12-min scans during the flare period.

Our flare occurs within the orbital phase window of the known optical brightenings, although it is unknown whether DQ Tau flared simultaneously in the optical. Using a $\mathrm{JD}_{0}$ value of $2449582.54 \pm 0.05$ and a period of $15.8043 \pm 0.0024$ days, as published in Table 2 of Mathieu et al. (1997), we calculate the phase of the flare peak to be 0.98 , or $\sim 7.6 \mathrm{~h}$ before closest approach. If we consider the error in the orbital period, propagated over 11 years, the flare in fact could have peaked anywhere between $25.4 \mathrm{~h}$ before periastron $(\Phi=0.93)$ to $11.0 \mathrm{~h}$ after $(\Phi=0.03)$, still well within the window of optical variability. Our other observations began at orbital phases of $-0.40,-0.09$, 0.11 , and 0.42 with respect to the same periastron encounter (corresponding to $-6.32,-1.42,1.74$, and 6.64 days to closest approach), but only the track nearest periastron $(\Phi=-0.02$ on 19 April) exhibits a brightening event. Therefore, we strongly suspect that the millimeter flare is connected to the binary motions, as well as to the optical variability, suggesting that this event is likely to be repeatable and even periodic.

\section{Discussion}

Of the three previous examples of flaring activity, DQ Tau draws the strongest parallel to V773 Tau A both in system geometry and flare properties. The similarities include binarity with a fairly equal mass distribution, high orbital eccentricity, and now an observed $3 \mathrm{~mm}$ flare that is nearly identical in rise time $(\sim 2 \mathrm{~h})$, decay time ( $\sim 5 \mathrm{~h}$, if we extrapolate in the case of DQ Tau), peak intensity $(\sim 400 \mathrm{mJy})$, incidence at periastron, and ratio $(\sim 25)$ of maximum intensity reached with respect to quiescent levels (see Massi et al. 2006, Fig. 2). The most significant difference is the lack of ongoing accretion around V773 Tau A as determined by its $\mathrm{H} \alpha$ equivalent width.

In the other examples, the coronal flare observed toward GMR-A produced millimeter activity for 13 days following its intial outburst. And while it achieved a similar peak flux after distance correction (see Furuya et al. 2003, Fig. 2), the outburst activity does not appear to be periodic (like in the case of V773 Tau A) since only a single radio flare was detected in the course of a 7-month monitoring campaign (Felli et al. 1993). As 
for ORBS, the radio flare shares a similar rise time and remains active several hours later when the track ended (see Forbrich et al. 2008, Fig. 1). Unfortunately, little more is known about the deeply embedded YSO or its flare activity. Further comparison between these scenarios and DQ Tau is difficult, given that we are unable to constrain the emission polarization or its extent.

Instead, from the millimeter perspective, the activity occurs within the window when overlapping magnetospheres from the binary components are expected to interact and produce flares like that observed on 19 April. And, in the case of V773 Tau A, similar star-star magnetospheric interactions were responsible for a nearly identical $3 \mathrm{~mm}$ flare profile. However, pulsed accretion flows in the DQ Tau system also show a periodicity coinciding with periastron. And while magnetospheric interaction is likely to explain the millimeter flare, the magnetospheres do not possess the energy required to power the optical brightenings with the frequency and duration observed at optical wavelengths (Mathieu et al. 1997).

DQ Tau also possesses an additional component, absent in the V773 Tau A system, which must be considered: a circumbinary disk. Increased accretion at periastron inherently requires inclusion of a star-disk interaction. When stars accrete, material is thought to be swept from the (circumbinary) disk, along the magnetic field lines, and onto the protostars (Shu et al. 1994). These field lines can be stretched or compressed during accretion events, changes that can also produce flares. Furthermore, in accretion theory, the balance between gas pressure and magnetic pressure often delineates the inner disk boundary. But when a stable accretion disk is disrupted (due to the dynamical motions of a binary) and not available to compress the stardisk field lines, radio flaring may occur more easily, and over larger distances, as the field lines roam more freely (Basri et al. 1997). Increased outflow signatures during optical brightenings are consistent with increased field alignment, if magnetic fields drive the outflows, and if pulsed accretion can organize the lines temporarily to power those outflows (Basri et al. 1997).

Because of this, the success with each orbit of a dynamicallydriven pulsed accretion mechanism could inhibit the incidence of millimeter activity (through compression of the field lines), resulting in an anti-correlation between optical and millimeter flaring. Alternatively, if the re-connection events provide a necessary pathway for accretion processes, then the haphazard nature of magnetic fields easily could explain the variability in the optical flaring (Mathieu et al. 1997) and the occurrence of millimeter flares and optical brightenings is correlated. Finally, the optical brightenings and the millimeter flares may be independent, only sharing the common periodicity of the binary orbit.

Simultaneous monitoring of this system at both optical and millimeter wavelengths is important for investigating whether magnetospheric and dynamical interactions between the stars around periastron are strongly related. Ultimately, core fragmentation during star formation may favor multiple-star systems like DQ Tau. Binaries comprise $65 \%$ or more of the stellar population in the middle of the main-sequence (Duquennoy \& Mayor 1991). And because planets have been detected around binaries (Eggenberger et al. 2004), there is an established need to study these systems and the impact of magnetospheric interactions and variable accretion processes on disk evolution.

\section{Conclusions}

The PMS spectroscopic binary, DQ Tau, has a highly eccentric orbit that should allow the stellar magnetospheres of the two components to interact at each periastron passage. We serendipitously observed evidence of this activity in a flare at $3 \mathrm{~mm}$, the first detected around a CTTS. The maximum intensity recorded $\left(355 \mathrm{mJy}\right.$ beam $\left.^{-1}\right)$ was 27 times the quiescent value $\left(13.3 \mathrm{mJy} \mathrm{beam}^{-1}\right)$. The observed peak occurred roughly $7.6( \pm 18.2) \mathrm{h}$ before periastron at $\Phi=0.98 \pm 0.05$, lasted several hours, and coincided with the average orbital phase $(\Phi=0.9)$ of the well-documented, periodic optical brightenings. Whether a simultaneous optical flare occurred is unknown.

The system's optical brightenings are consistent with the theory for pulsed accretion from the circumbinary disk onto the protostellar surfaces, regulated by the binary orbit, and perhaps assisted by magnetospheric interactions. The role of the magnetospheres during accretion events can now be studied observationally using methods established by Massi et al. (2006, 2008) in the analysis of V773 Tau A, the first example of coronae interactions between protostars where the authors were able to resolve the extent of the emitting region and the emission mechanism using very long baseline interferometry and polarization measurements. A similar observing program aimed at DQ Tau offers a unique opportunity to study star-disk interactions and re-connection events directly, and to test if magnetospheric interactions are necessary to facilitate periodic accretion.

Our findings also offer a small caution for millimeter flux points in SEDs that could contain unrecognized flare contributions if measured only once, with the time-consuming nature of observations at these wavelengths leading to a paucity of followup measurements.

Acknowledgements. We would like to thank Jin Koda and Joanna Brown for help with the data collection and reduction. We would also like to thank the referee for many valuable comments that have helped us to improve our manuscript. Support for CARMA construction was derived from the states of California, Illinois, and Maryland, the Gordon and Betty Moore Foundation, the Kenneth T. and Eileen L. Norris Foundation, the Associates of the California Institute of Technology, and the National Science Foundation. Ongoing CARMA development and operations are supported by the National Science Foundation under a cooperative agreement, and by the CARMA partner universities. The research of D.M.S. and M.R.H. is supported through a VIDI grant from The Netherlands Organization for Scientific Research.

\section{References}

Artymowicz, P., \& Lubow, S. H. 1996, ApJ, 467, L77

Basri, G., Johns-Krull, C. M., \& Mathieu, R. D. 1997, AJ, 114, 781

Beckwith, S., Sargent, A. I., Scoville, N. Z., et al. 1986, ApJ, 309, 755

Beckwith, S. V. W., \& Sargent, A. I. 1991, ApJ, 381, 250

Beckwith, S. V. W., Sargent, A. I., Chini, R. S., \& Guesten, R. 1990, AJ, 99, 924

Bower, G. C., Plambeck, R. L., Bolatto, A., et al. 2003, ApJ, 598, 1140

Duquennoy, A., \& Mayor, M. 1991, A\&A, 248, 485

Eggenberger, A., Udry, S., \& Mayor, M. 2004, A\&A, 417, 353

Feigelson, E. D., \& Montmerle, T. 1999, ARA\&A, 37, 363

Felli, M., Taylor, G. B., Catarzi, M., Churchwell, E., \& Kurtz, S. 1993, A\&AS, 101,127

Forbrich, J., Menten, K. M., \& Reid, M. J. 2008, A\&A, 477, 267

Furlan, E., Hartmann, L., Calvet, N., et al. 2006, ApJS, 165, 568

Furuya, R. S., Shinnaga, H., Nakanishi, K., Momose, M., \& Saito, M. 2003, PASJ, 55, L83

Hartigan, P., Edwards, S., \& Ghandour, L. 1995, ApJ, 452, 736

Hartmann, L., Hewett, R., \& Calvet, N. 1994, ApJ, 426, 669

Jensen, E. L. N., Dhital, S., Stassun, K. G., et al. 2007, AJ, 134, 241

Kaufmann, P., Correia, E., Costa, J. E. R., \& Zodi Vaz, A. M. 1986, A\&A, 157, 11

Massi, M., Menten, K., \& Neidhöfer, J. 2002, A\&A, 382, 152

Massi, M., Forbrich, J., Menten, K. M., et al. 2006, A\&A, 453, 959

Massi, M., Ros, E., Menten, K. M., et al. 2008, A\&A, 480, 489

Mathieu, R. D., Stassun, K., Basri, G., et al. 1997, AJ, 113, 1841

Najita, J., Carr, J. S., \& Mathieu, R. D. 2003, ApJ, 589, 931

Ohashi, N., Hayashi, M., Kawabe, R., \& Ishiguro, M. 1996, ApJ, 466, 317

Shu, F., Najita, J., Ostriker, E., et al. 1994, ApJ, 429, 781 